\title{
NORMAL, NOT PARACOMPACT SPACES
}

\author{
BY WILLIAM G. FLEISSNER ${ }^{1}$
}

\begin{abstract}
We describe some recently constructed counterexamples in general topology, including a normal, nonmetrizable Moore space, a normal para-Lindelöf, not paracompact space, and a normal, screenable, not paracompact space.
\end{abstract}

The period 1948-1952, when the notions of paracompactness and metrizability were investigated in terms of discrete and locally finite collections, was a period of great progress in point set topology. The work includes beautiful theorems, important counterexamples, and natural, unanswered questions. For example, Michael [M] showed that a space is paracompact (i.e. every open cover has a locally finite open refinement) if every open cover has a $\sigma$-locally finite open refinement. Can "locally countable" or " $\sigma$-disjoint" replace " $\sigma$-locally finite"? What if the space is normal? Bing's example B (see [B]) is a screenable, metacompact Moore space which is not paracompact. Is there such an example which is normal? The purpose of this announcement is to describe the series of papers $\left[\mathbf{F}_{1}, \mathbf{N}, \mathbf{F}_{2}, \mathbf{F}_{3}, \mathbf{R}\right]$ which answer the above and similar questions by constructing normal, not paracompact spaces.

Let us review some definitions. In this paper we consider only regular, $T_{3}$ spaces. A collection of subsets of a space $X$ is locally finite (resp. locally countable) if every $x \in X$ has a neighborhood meeting finitely many (resp. countably many) elements of the collection. A collection has the $\sigma$-property if it is the union of countably many collections with the property. A space is screenable (resp. paraLindelöf) if every open cover has a $\sigma$-disjoint (resp. locally countable) open refinement. A More space is a special type of first countable space; we will not need the precise definition.

We begin by describing the nonseparable metric space, $F$, from which the spaces are constructed. Points of $F$ are functions from $\omega$ (the set of natural numbers) to $\omega_{1}$ (the set of countable ordinals). The distance, $d(f, g)$ between two points of $F$ is $2^{-n}$, where $n$ is least such that $f(n) \neq g(n)$.

Let $\Sigma_{n}$ be the set of functions to $\omega_{1}$ with domain $\{0,1,2, \ldots, n-1\}$. For $\sigma \in \Sigma_{n}$ we define $N_{\sigma}=\{f \in F: \sigma \subset f\}=\{f \in F: f(0)=\sigma(0), \ldots$, $f(n-1)=\sigma(n-1)\}$. Then $\left\{N_{\sigma}\right.$ : for some $\left.n \in \omega, \sigma \in \Sigma_{n}\right\}$ is a base for $F$. We

Received by the editors January 5, 1982.

1980 Mathematics Subject Classification. Primary 54D18, 54E30.

Key words and phrases. Normal, paracompact, metrizable, para-Lindelöf, screenable, normal Moore space, counterexamples in topology.

1 Partially supported by NSF Grant MCS-79-01848. 
say a pair $\{\rho, \tau\}$ of elements of $\Sigma_{n}$ are entwined if $\rho(0)<\tau(0)<\rho(1)<\tau(1)<$ $\cdots<\rho(n-1)<\tau(n-1)$. Let $Q_{1}$ be the set of entwined pairs. The point set of $X_{1}$, the space of $\left[\mathrm{F}_{1}\right]$ is $F \cup Q_{1}$. Points of $Q_{1}$ are isolated. Basic open neighborhoods of points in $F$ have the form $B(\sigma, a)=N_{\sigma} \cup\left\{\left\{\sigma^{\prime}, \nu\right\}: \sigma \subset \sigma^{\prime}\right.$ and $v(0) \notin a$ \} where $\sigma \in \Sigma_{n}$ for some $n$ and $a$ is a finite subset of $\omega_{1} . X_{1}$ was the first example of a nonmetrizable space with a $\sigma$-locally countable base.

The notion of full set is the key to show that $X_{1}$ is neither normal nor para-Lindelöf. We call a subset $S$ of $\Sigma_{n}$ full if for all $i<n$ and $\sigma \in S$ the set $\left\{\sigma^{\prime}|i+1: \sigma| i \subset \sigma^{\prime} \mid i+1, \sigma^{\prime} \in S\right\}$ is uncountable. (The picture in our mind is that $S$ is the top, $n$ th, level of a tree. A node at the $i$ th level is an element of $\Sigma_{i}$, and, if $i<n$, has uncountably many extensions at the next level.) The combinatorics of full sets are summarized in the following lemma.

Lemma 1. (a) If $\bigcup\{[\sigma]: \sigma \in T\}=F$, then for some $n, T \cap \Sigma_{n}$ has $a$ full subset.

(b) If $S$ is full and $h$ is a function from $S$ to a countable set, then $h$ is constant on a full subset of $S$.

(c) If $S$ is full, then there is an infinite subset of $S$ all of whose pairs are entwined.

Next we consider Navy's space $X_{2}$ (see [N]). Intuitively, the idea is to replace each point of $Q_{1}$ with a copy of Bing's Example $\mathrm{G}$ (see [B]). Formally, let $Q_{2}$ be the set of triples $\langle\rho, \tau, g\rangle$ where $\{\rho, \tau\}$ is entwined and $g$ is a function from the open sets of $F$ to $\{0,1\}$. The point set of $X_{2}$ is $F \cup Q_{2}$; again points of $Q_{2}$ are isolated. Basic open neighborhoods of points of $F$ have the form $B(\sigma, a)=N_{\sigma} \cup G(\sigma, a)$, where $G(\sigma, a)=\{\langle\rho, \tau, g\rangle:$ (i) $\sigma \subset \rho$ or $\sigma \subset \tau$, (ii) for all $U \in a \mid g(U)=1$ iff $\left.N_{\sigma} \subset U\right\}$. Here $\sigma \in \Sigma_{n}$ for some $n$ and $a$ is a finite set of open sets of $F$ satisfying $N_{\sigma} \subset U$ or $N_{\sigma} \cap U=\varnothing$. A short investigation shows that normality is "built in"; para-Lindelöf follows quickly. That $X_{2}$ is not paracompact follows from full set combinatorics.

$X_{2}$ is far from being first countable, much less a Moore space; points of $F$ require $2^{\aleph_{1}}$ neighborhoods for a base because there are so many open subsets of $F$. At the cost of complicating the proof of normality, the character can be reduced to $2^{\aleph_{0}}$ by restricting the domain of the g's to countable unions of basic open sets. A nonmetrizable normal Moore space, $X_{3}$, can be constructed by assuming the Continuum Hypothesis. The countable unions of basic open sets can be well ordered type $\omega_{1}$. Let $Q_{3}$ be the set of triples $\langle\rho, \tau, g\rangle$ where $\rho, \tau$ are entwined and $g$ is a function from an initial segment (depending on $\rho$ ) of this well-ordering to $\{0,1\}$. The definition of the basic open sets requires some technical details, and so is omitted. The proof of normality splits into two cases. For 
"fast functions" the argument is as for $X_{2}$. For "slow functions" it is convenient to introduce for each countable limit ordinal, $\delta$, an increasing simple sequence $\left(\delta_{i}\right)_{i \in \omega}$ converging to $\delta$. An important property of these sequences is

LEMMA 2. For each $\beta<\omega_{1}$, there is a function $m_{\beta}$ from the limit ordinals less than $\beta$ to $\omega$ such that for distinct $\delta, \eta$ there is $i<\max \left\{m_{\beta}(\eta), m_{\beta}(\delta)\right\}$ such that $\delta_{i} \neq \eta_{i}$.

So far, we have ignored the post-Bing results on the normal Moore space conjecture (see [T]). Now we must consider Nyikos' "provisional solution to the normal Moore space problem" $\left[\mathbf{N}_{\mathbf{y}}\right]$. Nyikos showed that it followed from the Product Measure Extension Axiom that all normal Moore spaces are metrizable. The qualifier "provisional" was added because a large cardinal was used to show this axiom consistent. We complete this solution by showing that a large cardinal is necessary. Define $\beth_{i}$ inductively: $\beth_{0}=\aleph_{0} ; \beth_{i+1}=2^{\beth}$; set $\kappa=$ $\sup \left\{\beth_{i}: i \in \omega\right\}$. It follows from work of Jensen that either there is an inner model with a measurable cardinal or $2^{\kappa}=\kappa^{+}$and there is a special subset of $\kappa^{+}$ for which an analog of Lemma 2 holds. In the latter case we can construct a nonmetrizable normal Moore space $X_{4}$ in the same way we constructed $X_{3}$.

Spaces $X_{1}-X_{4}$ form a development of certain techniques for constructing topological spaces. Although not part of that series, Rudin's normal, screenable, nonparacompact space, $X_{5}$, shares many of the ideas and techniques. Points of $X_{5}$ are carefully chosen functions from $\omega \times(\omega-n)$ to $\omega_{1}$ (i.e. the domain is a doubly infinite matrix with the bottom cut off. For each $n$, there are many such functions). Entwined pairs occur implicitly in the proof; full sets appear explicitly. The functions are carefully chosen using $\diamond^{++}$, a consequence of $V=L$, Gödel's axiom of constructibility. In $\left[\mathbf{F}_{4}\right] \diamond^{++}$was derived from $V=L$ in order to construct a first countable, normal, not collectionwise normal space. While this construction turned out to be tangential to the solution of the normal Moore space problem, $\diamond^{++}$provided the combinatorics Rudin needed to construct $X_{5}$.

Watson [W] has used these techniques to answer the last open questions about $\sigma$-locally countable bases.

\section{BIBLIOGRAPHY}

[B] R. H. Bing, Metrization of topological spaces, Canad. J. Math. 3 (1951), 175-186.

[ $\left.\mathrm{F}_{1}\right]$ W. G. Fleissner, A collectionwise Hausdorff, nonnormal Moore space with a $\sigma$ locally countable base, Topology Proc. 4 (1979), 83-96.

$\left[\mathrm{F}_{2}\right]-$ Normal Moore spaces, continuum hypothesis and large cardinals, Proc. Nat. Acad. Sci. U. S. A. (to appear).

[ $\left.\mathrm{F}_{3}\right]-$ If all normal Moore spaces are metrizable, then there is an inner model with a measurable cardinal, Trans. Amer. Math. Soc. (to appear).

$\left[\mathrm{F}_{4}\right] \longrightarrow$ Son of George and $V=L$, J. Symbolic Logic (to appear).

[M] E. Michael, A note on paracompactness, Proc. Amer. Math. Soc. (1953), $831-$ 838.

[N] C. Navy, Para-Lindelöf versus paracompact, Topology Appl. (to appear). 
[ $\mathrm{N}_{\mathrm{y}}$ ] P. J. Nyikos, A provisional solution to the normal Moore space problem, Proc. Amer. Math. Soc. 78 (1980), 429-435.

[R] M. E. Rudin, A normal screenable, not paracompact space, Topology Appl. (to appear).

[T] F. D. Tall, The normal Moore space problem, Math. Centre Tracts 116 (1979), 263-270.

[W] W. S. Watson, Spaces with o-locally countable bases (to appear).

DEPARTMENT OF MATHEMATICS, UNIVERSITY OF PITTSBURGH, PITTSBURGH, PENNSYLVANIA 15260 